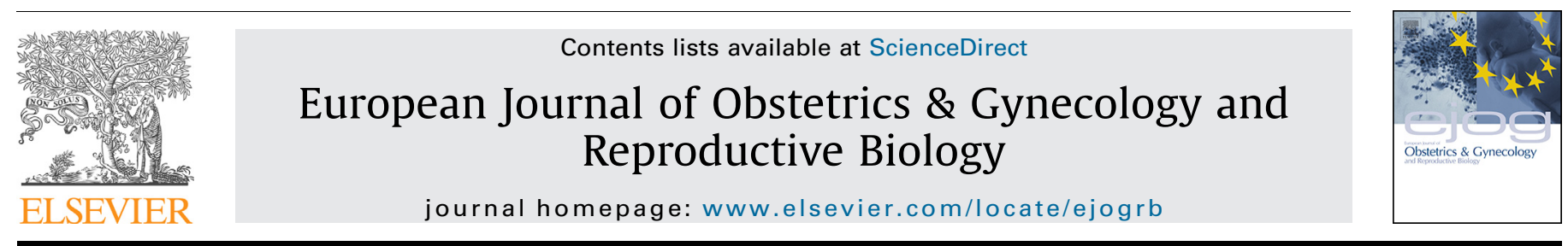

Correspondence

\section{Can manual lymph drainage be improved or not: That's the question! A response letter}

\section{Dear Editor-in-Chief,}

We have read the comments on the recently published protocol of the EFforT-BCRL trial [1] with great interest. The aim of this multi-centre randomized controlled trial (RCT) was to determine the effectiveness of fluoroscopy-guided manual lymph drainage (MLD) in addition to the other components of decongestive lymphatic therapy and compared to traditional or placebo MLD, in the treatment of breast cancer-related lymphoedema. The protocol of this trial adheres to the recommended CONSORT guidelines and the work received substantial contributions from all stakeholders in order to meet the required quality standards.

In our opinion, the concerns voiced in the comments are unjustified and we would like to address three issues in this response letter.

First, with regard to the following comment: "Their 'traditional' $M L D$ (in their group B) seems to be what they do themselves but, neither what many other Belgian physiotherapists do routinely, nor what is done in other countries. Indeed, they write that manoeuvers "to create resorption of lymph" are part of their "new" MLD but are not mentioned when they describe what they define as "traditional" MLD. Unfortunately for the authors, these manoeuvers are part of the MLD for instance taught by the Földi's and Leduc's schools. Thus, the conclusions of their study could not be used to omit MLD taught by these schools."

It is correct that many schools train physiotherapists in MLD (e.g. Lerner, Casley-Smith, Leduc, Vodder, and colleagues). This makes it difficult to develop a method that includes all MLD methods, a so-called traditional method. In order to define this traditional method of MLD, consensus was reached after discussion with a team of experts in the field of lymphology with manifold years of experience in MLD according to the Leduc and the Vodder method. We have opted for a combination of these two methods because 1) the effects of these methods were investigated in the various RTCs reported in the meta-analysis of Huang et al [2] and systematic review of Ezzo et al [3], and 2) these two methods are most frequently applied in Belgium.

In contrast to what was stated by Pierre Bourgeois in his letter we use the term 'traditional method of MLD' to refer to a technique applied to stimulate resorption of the lymph by the lymph capillaries. The technique is performed with the whole hand whereas in the fluoroscopy-guided MLD only the thumbs are used (to create higher pressure).

Second, the value of future conclusions resulting from this trial was questioned because of the following statement: "if results would reveal that the fluoroscopy-guided MLD yields a better outcome compared to the traditional MLD and/or the placebo MLD, one does still not know whether this is due to the knowledge of the patientspecific lymphatic anatomy, or due to the altered MLD manoeuvres".

In our opinion, it is incorrect to state that the protocol will not allow any valuable conclusion. The aim of our study was to investigate the effect of a completely optimized MLD technique (i.e. optimization of the MLD manoeuvres whose physiological effects after one session of fluoroscopy-guided MLD have already been proven [4,5], as well as having knowledge of the patientspecific lymphatic network). In case this technique proves to be more effective, a new study should be designed to investigate why it is more effective: because of the manoeuvres or because of the knowledge of the lymphatic network. Additionally, given the facts that the more recently published RCTs investigating the added value of MLD failed to prove an additional effect [6,7], and that the Cochrane systematic review only showed an additional effect of $7 \%$ [3], there is a valid reason to question this added value and to further investigate it. Therefore, we considered it important to also have the ability to compare results with those of a group receiving placebo MLD, in order to investigate whether MLD has an additional effect or not.

Third, four rather unspecified difficulties were raised.

- It is mentioned that we are "using multi-layer bandaging with component different from the one used by other physiotherapists" as cited in the comment. However, we apply this type of multi-layer bandaging as a standard treatment since it is based on the available evidence found in literature. Additionally, this method of bandaging is recommended by the International Compression Club (ICC), as it is also the applied technique in the 3 expertise centres in Belgium (UMC St-Pierre University Hospital Brussels, University Hospitals Leuven, and CHU UCL Godinne University Hospital Namur). Nevertheless, in case of any scientific evidence against the use of this technique, we would be delighted to learn from it. Unfortunately, we were unable to find any supporting references of RCTs recommending the use of one technique over another.

- It is correct that the two physiotherapists LV and TDV do not work in the department of rehabilitation of the St-Pierre University Hospital, nor in one of the other participating centres. However, both therapists are specialised in the field of lymphology and consequently in the applied decongestive lymphatic therapy as well. For this reason, and based on the approval by the Ethical Committee of the University Hospitals of Leuven (main Ethical Committee) and the positive advice from the Ethical Committees of all other participating centres (CME reference S58689, EudraCT Number 2015-004822-33), both therapists perform the treatments in all study centres.

- The reason why "the use of specific systems for the volumetric and perimetric evaluations" is mentioned as a difficulty is not entirely clear to us. Unfortunately, the reason why such a statement is made, is lacking. Reliability of circumference measurements 
using a perimeter has been proven [8], and water displacement is currently considered as the golden standard [9] because of its very good clinimetric properties to quantify arm volume [10-12]. Moreover, a study to investigate reliability of the applied water displacement method in the EFforT-BCRL trial has been conducted and will be published shortly $[13,14]$.

- The lymphofluoroscopic investigations are used to determine the patient-specific superficial lymphatic architecture. The authors will further investigate reproducibility of this imaging technique (in the same way as they have investigated reproducibility of lymphoscintigraphy in 2014 [15]), with the aim to provide an appropriate, evidence-based answer to this important question in due course.

We hope to have offered elucidating answers to the concerns voiced in the previous letter to the editor.

\section{References}

[1] De Vrieze T., Vos L, Gebruers N, et al. Protocol of a randomised controlled trial regarding the effectiveness of fluoroscopy-guided manual lymph drainage for the treatment of breast cancer-related lymphoedema (EFforT-BCRL trial). Eur J Obstet Gynecol Reprod Biol 2018;221:177-88, doi:http://dx.doi.org/10.1016/j. ejogrb.2017.12.023 [published Online First: Epub Date].

[2] Huang TW, Tseng SH, Lin CC, et al. Effects of manual lymphatic drainage on breast cancer-related lymphedema: a systematic review and meta-analysis of randomized controlled trials. World J Surg Oncol 2013;11:15, doi:http://dx.doi. org/10.1186/1477-7819-11-15 [published Online First: Epub Date].

[3] Ezzo J, Manheimer E, McNeely ML, et al. Manual lymphatic drainage for lymphedema following breast cancer treatment. Cochrane Database Syst Rev 2015;(5)Cd003475, doi:http://dx.doi.org/10.1002/14651858.CD003475.pub2 [published Online First: Epub Date].

[4] Belgrado JP, Vandermeeren L, Vankerckhove S, et al. Near-infrared fluorescence lymphatic imaging to reconsider occlusion pressure of superficial lymphatic collectors in upper extremities of healthy volunteers. Lymphat Res Biol 2016;14(2)70-7, doi:http://dx.doi.org/10.1089/lrb.2015.0040 [published Online First: Epub Date].

[5] Tan IC, Maus EA, Rasmüssen JC, et al. Assessment of lymphatic contractile function after manual lymphatic drainage using near-infrared fluorescence imaging. Arch Phys Med Rehabil 2011;92(5)756-64, doi:http://dx.doi.org/ 10.1016/j.apmr.2010.12.027 e1 [published Online First: Epub Date].

[6] Bergmann A, da Costa Leite Ferreira MG, de Aguiar SS, et al. Physiotherapy in upper limb lymphedema after breast cancer treatment: a randomized study. Lymphology 2014;47(2):82-91.

[7] Gradalski T, Ochalek K, Kurpiewska J. Complex decongestive lymphatic therapy with or without vodder II manual lymph drainage in more severe chronic postmastectomy upper limb lymphedema: a randomized noninferiority prospective study. J Pain Symptom Manage 2015;50(6)750-7, doi:http:// dx.doi.org/10.1016/j.jpainsymman.2015.06.017 [published Online First: Epub Date].

[8] Devoōgdt N, Lemkens H, Geraerts I, et al. A new device to measure upper limb circumferences: validity and reliability. Int Angiol 2010;29(5):401-7.

[9] The diagnosis and treatment of peripheral lymphedema. Consensus document of the International society of lymphology. Lymphology 2013;46(1):1-11.

[10] Gebruers N, Truijen S, Engelborghs S, De Deyn PP. Volumetric evaluation of upper extremities in 250 healthy persons. Clin Physiol Funct Imag 2007;27(1)
17-22, doi:http://dx.doi.org/10.1111/j.1475-097X.2007.00708.x [published Online First: Epub Date].

[11] Damstra RJ, Glazenburg ËJ, Hop WC. Validation of the inverse water volumetry method: a new gold standard for arm volume measurements. Breast Cancer Res Treat 2006;99(3)267-73, doi:http://dx.doi.org/10.1007/s10549-0069213-0 [published Online First: Epub Date].

[12] Deltombe T, Jamart J, Recloux S, et al. Reliability and limits of agreement of circumferential, water displacement, and optoelectronic volumetry in the measurement of upper limb lymphedema. Lymphology 2007;40(1):26-34.

[13] Evaluation of the reliability of four measuring methods of hand's perimeter and volume: buoyancy forces valgrado system, circumference measurement, figure-of-eight method and Manu3Metrix scanner. $8^{\text {Th }}$ International Lymphoedema Framework Conference. .

[14] T. De Vrieze, N. Gebruers, A. De Groef, L. Dams, E. Van der Gucht, I. Nevelsteen, N. Devoodt. Reliability and time efficiency of five different methods to determine arm volume in patients with breast cancer-related lymphoedema. In publication.

[15] Devoogdt N, Van den Wyngaert T, Bourgeois P, et al. Reproducibility of lymphoscintigraphic evaluation of the upper limb. Lymphat Res Biol 2014;12 (3)175-84, doi:http://dx.doi.org/10.1089/lrb.2013.0034 [published Online First: Epub Date].

Tessa De Vrieze $\mathrm{a}^{\mathrm{a}, \mathrm{b}}$

${ }^{a} K U$ Leuven - University of Leuven, Department of Rehabilitation Sciences, Leuven, Belgium

${ }^{b}$ University of Antwerp, Department of Rehabilitation Sciences and Physiotherapy, MOVANT, Antwerp, Belgium

Nick Gebruers ${ }^{\mathrm{a}, \mathrm{b}}$

${ }^{a}$ University of Antwerp, Department of Rehabilitation Sciences and Physiotherapy, MOVANT, Antwerp, Belgium

${ }^{b}$ University of Antwerp \& Antwerp University Hospital, Multidisciplinary Oedema Clinic, Antwerp, Belgium

Nele Devoogdt ${ }^{\mathrm{a}, \mathrm{b}, *}$

${ }^{a}$ KU Leuven - University of Leuven, Department of Rehabilitation Sciences, Leuven, Belgium

${ }^{b}$ UZ Leuven - University Hospitals Leuven, Department of Vascular Surgery and Department of Physical Medicine and Rehabilitation, Centre for Lymphoedema, Leuven, Belgium

* Corresponding author at: Centre for Lymphoedema, University Hospitals Leuven, Department of Rehabilitation Sciences, KU Leuven, O\&N 4 Herestraat 49, 3000 Leuven, Belgium. E-mail address: nele.devoogdt@kuleuven.be (N. Devoogdt).

Received 4 July 2018

Available online xxx 